\title{
Autoantibodies Profile in Vitiligo
}

\section{Kabir Magaji Hamid ${ }^{1,2 *}$, Zelalem Kiros Bitsue ${ }^{2}$ and Abbas Mirshafiey}

${ }^{1}$ Department of Immunology, Faculty of Medical Laboratory Sciences, Usmanu Danfodiyo University Sokoto, P.M.B 2346, Nigeria

${ }^{2}$ Department of Immunology, School of Public Health, Tehran University of Medical Sciences-International Campus (TUMS-IC) Tehran-14155, Box: 6446, Iran

${ }^{3}$ Department of Immunology, School of Public Health, Tehran University of Medical Sciences, (TUMS) Tehran-14155, Box: 6446, Iran

\begin{abstract}
Vitiligo is one of the disease which is yet to understand its pathogenesis, however many studies associate this disease as an autoimmune. Detection of autoimmune cells in the serum, lesional and perilesional area of vitiligo patients gives more insight on the disease mechanism. Presence of autoantibodies against melanocytes antigens in vitiligo patients indicates an autoimmune involvement in the aetiology of the disease. Identification and characterization of vitiligo autoantibodies would pave the way for developing new laboratory test for diagnosis. Studying the autoantibodies profile can give an impression on the disease condition of vitiligo patients. We realized the need of research emphasis in this area as more is yet to be discovered. In this review we give an account on different autoantibodies and their associated autoantigens in vitiligo as another effort of providing an updated data for detail analysis.
\end{abstract}

Keywords: Antibodies; Autoimmunity; Melanocytes; Pigmentation; Vitiligo

\section{Introduction}

Vitiligo is an acquired depigmenting disorder in which melanocytes are destroyed, resulting in patchy depigmentation on skin and mucosal surfaces [1,2]. The worldwide prevalence is range from $0.5-2 \%[3]$. Clinically, vitiligo presents as round or oval white, hypopigmented macules with regular or raised red borders [4]. The disease was classified based on distribution patterns of vitiliginous lesions into focal vitiligo (isolated lesion), segmental vitiligo (unilateral macular lesions which generally cover a dermatome), non-segmental (generalized) vitiligo (most common form, disseminated macules of variable size, usually with a symmetric distribution and a certain predilection for extensor surfaces) [5,6] and universalis vitiligo (severe form that affects more than $80 \%$ of the body surface) [4].

The pathogenesis of vitiligo is unclear. although both genetic [7] and environmental factors are the ones implicated as the major cause [8], however, there are other several factors proposed in the pathogenesis of the disease (Figure 1), these include the following, physical trauma $[9,10]$, psychological stress [11], infections [12], neural factors $[11,13,14]$, biochemical factors [15-17], melanocytes growth factors [18], melanocortin hormones [19] and autoimmunity [20].

Most authorities favored the autoimmune causes due to the strong associations of vitiligo with multiple autoimmune diseases; the presence of autoantibodies [21,22] (Table 1), so that autoreactive T lymphocytes against pigment cells supports the theory that there is an autoimmune involvement in the aetiology of the disease [23]. However, even if the specific antibodies to pigment cells or secondary antibodies are not pathogenic, the identification and characterization of their target antigens could be a landmark for uncovering the pathogenic mechanism, formation of autoantibodies [24] and development of biomarkers. In this review we describe some autoantibodies and their associated autoantigens as potential biomarkers for laboratory diagnosis, treatment, monitoring and assessment of vitiligo.

\section{Anti-Melanocytes}

Melanocytes originate from neural crest and are responsible for the synthesis of melanin in melanosomes, membrane-bound organelles $[25,26]$. They are able to secrete a wide range of signal molecules, including cytokines, Pro-opiomelanocortin (POMC) peptides, catecholamines, and Nitric oxide in response to UV irradiation and other stimuli, for the regulation of variety of skin cells [27]. In active non-segmental vitiligo, melanocyte cytotoxicity is associated with increase in serum levels of immunoglobulin G (IgG) antimelanocyte/vitiligo antibodies (V-IgG) and immunologic markers [28]. IgG anti-melanocyte antibodies were reported to induce melanocyte damage in vitro by a complement-mediated mechanism and antibodydependent cellular cytotoxicity [29,30]. The melanocytotoxicity could be due to wrong presentation of vitiligo antigens to destructive cytotoxic T cells; this result from abnormal expressions of HLADR and increase expression of intercellular adhesion molecule-1 (ICAM-1) on melanocytes by IgG anti-melanocyte antibodies [29,31]. Antibodies to melanocytes occur at a significantly increased frequency in the sera of vitiligo patients when compared with healthy individuals [32]. Interestingly, correlations can also exist between the incidence and level of melanocyte antibodies and both the activity and extent of vitiligo [33]. Identification of anti-melanocyte or vitiligo antibodies against target antigens is useful in developing new diagnostic tests and serves as biomarkers for assessing the progress of the disease [23].

\section{Anti-thyroid peroxidase (anti-TPO)}

Anti-TPO antibodies are specific for the autoantigen TPO; found in active phase of chronic autoimmune thyroiditis; can be used in monitoring the disease progress in patients with these antibodies [34]. Most of the anti-TPO antibodies are produced by thyroid infiltrating lymphocytes and partly from lymph nodes and bone marrow [35]. In the past decades, many research teams reported the associations between vitiligo and other autoimmune diseases such as thyroid disease and anti-thyroid antibodies [36]. A previous study by Dave et al. reported $31.4 \%$ prevalence of thyroid-specific autoantibodies in patients with vitiligo [37]. A more recent study reported a mean prevalence of $20.8 \%$ in patients with vitiligo [38]. Again, KasumagicHalilovic et al. found higher frequency of anti-TPO in vitiligo patients than control group [39]. Considering these findings vitiligo shows strong association with thyroid autoimmunity, therefore anti-TPO

*Corresponding author: Kabir Magaji Hamid, Department of Immunology, Faculty of Medical Laboratory Sciences, Usmanu Danfodiyo University Sokoto P.M.B 2346, Nigeria, Tel: +989102159812; E-mail:kmhamid@hotmail.co.uk

Received April 14, 2015; Accepted May 15, 2015; Published May 25, 2015

Citation: Hamid KM, Bitsue ZK, Mirshafiey A (2015) Autoantibodies Profile in Vitiligo. Pigmentary Disorders 2: 187. doi:10.4172/2376-0427.1000187

Copyright: ( $) 2015$ Hamid KM, et al. This is an open-access article distributed under the terms of the Creative Commons Attribution License, which permits unrestricted use, distribution, and reproduction in any medium, provided the original author and source are credited. 


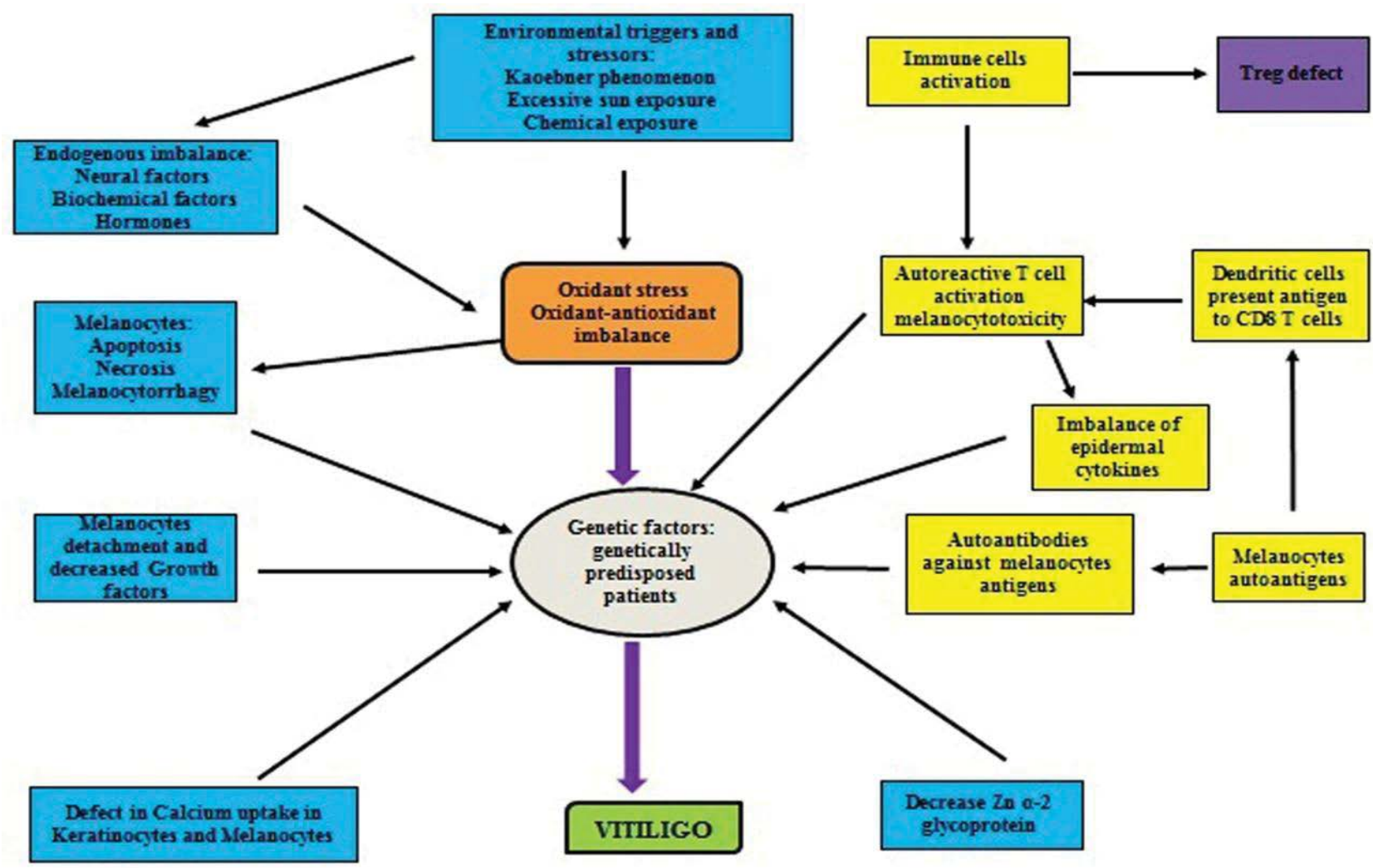

Figure 1: A schematic representation of proposed factors in vitiligo aetiology. The factors implicated in aetiology of vitiligo interacts either synergistically or independently to induce the destruction of melanocytes. Activation of immune cells results in proliferation and differentiation of autoreactive T cells leading to melanocytotoxicity and secretion of proinflammatory cytokines in epidermis. Dendritic cells present melanocytes antigens to autoreactive, anti-melanocytes CD8 T cells. Autoantibodies against the melanocytes antigens, likely generated due to melanocyte damage, possibly destroy the pigment cells by complement activation and/or ADCC. Defect in number and function of regulatory T cells may promote the loss of control of the immune cells. Genetic predisposition, metabolic deregulation together with impaired redox status, environmental aggressions and neural dysfunction probably act in an interrelated way to trigger local inflammation and damage of cells. Other factors may likely contribute in loss of melanocytes in genetically predisposed patients.

\begin{tabular}{|c|c|c|}
\hline Antibody reactivity & $\begin{array}{c}\text { Number of patients with } \\
\text { antibodies (\%) }\end{array}$ & Reference \\
\hline Gastric parietal cells & $6 / 20(30)$ & {$[61]$} \\
\hline Thyroid cytoplasm & $22 / 80(28)$ & {$[64]$} \\
\hline Thyroid peroxidase & $10 / 20(50)$ & {$[61]$} \\
\hline Thyroglobulin & $8 / 20(40)$ & {$[61]$} \\
\hline Tyrosinase & $5 / 46(10.9)$ & {$[65]$} \\
\hline TYRP1 & $8 / 84(9.5)$ & {$[24]$} \\
\hline TYRP2 & $20 / 30(67)$ & {$[60]$} \\
\hline Adrenal gland & $3 / 80(4)$ & {$[64]$} \\
\hline Pancreatic islet cells & $7 / 96(7.2)$ & {$[36]$} \\
\hline Anti-nuclear antibody & $4 / 55(7.3)$ & {$[32]$} \\
\hline IgM-rheumatoid factor & $6 / 55(10.8)$ & {$[32]$} \\
\hline Lamin A & $24 / 84(28.6)$ & {$[24]$} \\
\hline MCHR1 & $24 / 145(16.55)$ & {$[66]$} \\
\hline Y-enolase & $4 / 53(8)$ & {$[67]$} \\
\hline a-enolase & $5 / 53(9)$ & {$[67]$} \\
\hline HSP90 & $7 / 53(13)$ & {$[67]$} \\
\hline HSP70 & $16 / 60(26)$ & {$[68]$} \\
\hline Fibrin beta & $16 / 60(26)$ & {$[68]$} \\
\hline Osteopontin & $5 / 53(9)$ & {$[67]$} \\
\hline Ubiquitin-conjugating enzyme & $8 / 53(15)$ & {$[67]$} \\
\hline Translation-initiation factor 2 & $3 / 53(6)$ & {$[67]$} \\
\hline Tabl & & \\
\hline
\end{tabular}

Table 1: Some of the antibodies detected in vitiligo patients. detection in vitiligo patients could be a useful markers for assessment of the disease. In this case more in-depth studies are required to give detail information.

\section{Anti-melanocortin 1 receptor $(\mathrm{MC} 1 \mathrm{R})$}

The melanocortin peptide a-melanocyte-stimulating hormone (MSH) is an important regulatory agent in skin pigmentation, inflammatory modulation and response to stress $[19,40]$. The $\alpha-\mathrm{MSH}$ binds to MC1R on the melanocyte [41] to increase tyrosinase activity and eumelanin production; this action could lead to regulation of melanocytes, skin pigmentation, Nitric oxide production and release of other signalling molecules from melanocytes [41]. Moreover, Pichler et al. reported that $\alpha-\mathrm{MSH}$ levels were significantly lower in vitiligo patients compared to normal individuals [42]. Since the expression level of $a-\mathrm{MSH}$ in the melanocytes from lesion and perilesion area of vitiligo skin are lower than that from normal skin [43]. The proportion of a-MSH immuno-positive melanocytes is significantly reduced in the lesional and perilesional skin of vitiligo patients compared to controls [44]. Autoantibodies against MC1R are rare or absent in sera of vitiligo patients [45].

\section{Melanin concentrating hormones receptor (MCHR)-binding antibodies}

MCHR1has been identified as a B cell autoantigen in vitiligo; the 
antibodies against this receptor are detected in binding and functionblocking assays [46]. A previous study has shown they can block the stimulation of the receptor by $\mathrm{MCH}$ in a heterologous cell line [47]. Noteworthy, the $\mathrm{MCH} / \mathrm{MCHR}$ signalling pathway is critical in regulating melanocyte function along with $\alpha-\mathrm{MSH}$ and the MC1R [48], any action by MCHR1 antibodies to interfere in the normal functioning of the receptor could change the pigment cell behavior. However, the effect of these antibodies on the MCHR1 in melanocytes either in vitro or in vivo is obscure [23]. Few years back, the technique of peptide phage-display has identified the MCHR1 as a target of vitiligo patient antibodies [49]. Moreover, MCHR autoantibodies were found at a significantly increased frequency in the vitiligo patient group compared to healthy controls. In addition, vitiligo patient IgGs were tested for MCHR autoantibodies that could mediate antibody-dependent cellmediated cytotoxicity via the receptor [47]. More study on MCHR1 antibodies is required to affirm their role in vitiligo aetiology and could be reliable biomarkers for vitiligo diagnosis in future.

\section{Melanoma antigen recognized by $\mathrm{T}$-cells (MART)}

MART is a protein antigen that is found on the surface of melanocytes. Antibodies against this antigen are used in the medical specialty of anatomic pathology in order to recognize cells of melanocytic differentiation, useful for the diagnosis of a melanoma [4]. A study showed that serum level of melanocyte antibody in children with vitiligo was significantly higher than that in normal subjects and MART-1 was found binding to specific MART-1 antibody [50]. This indicates that MART-1 antibody may be useful in monitoring the autoimmune mechanism in patients with vitiligo. However, Bam and Bagchi reported that MART-1 transcript is not detected in the peripheral blood mononuclear cells of vitiligo patients but is detected in healthy controls [51]. The non-expression of MART1 may be plausibly implicated for the tolerance breakdown and subsequent induction of autoimmunity in the vitiligo patients. Antibody against MART-1 requires further investigation, as it may potentially give an insight on autoimmune mechanism in vitiligo patients.

\section{Lamin A (VIT 75) autoantibody}

Vitiligo autoantigen VIT75 is identified as Lamin A in Vitiligo. VIT75 is melanocyte membrane antigen that had been observed, but not identified until recently, indeed, its immunopathogenic role in vitiligo remains unknown [24]. However, anti-lamin antibodies are detected in sera in a range of autoimmune diseases [52]; although the process on how these antibodies are induced in vivo is yet to be clear. Interestingly, majority of vitiligo patients with anti-lamin A had at least one autoimmune disorder; the prevalence rate and titers of the antibodies are higher than in the patients without autoimmune disease or healthy controls [24]. Furthermore, previous study showed that $83 \%$ of vitiligo patients had the antibodies against VIT75 while only $7 \%$ of controls showed reactivity against it [53]. Henceforth, antilamin A antibody may likely be a potential marker of non-segmented vitiligo with autoimmune disease [24]. Here the use of serological proteome analysis to target autoantibodies in vitiligo patients is highly encouraged to give more insight.

\section{Tyrosine Hydroxylase (TH) antibody}

$\mathrm{TH}$ was generally accepted as autoantigen in vitiligo using phagedisplay technology [54]. TH was suggested to play role in melanin synthesis by supplying a substrate L-dopa for tyrosinase which subsequently converts L-tyrosine to dopaquinone, a precursor of melanin [55]. Although there are controversies on the presence of $\mathrm{TH}$ in melanocytes, existing knowledge indicates that the TH mRNA has been found in human epidermal melanocytes and this enzyme is located on the melanosomal membrane together with tyrosinase. In contrast, Kagedal et al. reported that the levels of TH mRNA in several melanocytes were insignificant or undetectable [56]. However, $\mathrm{TH}$ antibodies were elevated in patients with active vitiligo and the antibodies were reported to target $\mathrm{TH}$ in non-segmental vitiligo [54]. Also Kemp and his co-workers reported a significant increase in the prevalence of $\mathrm{TH}$ antibodies in patients with non-segmental vitiligo when compared with controls [54]. In addition, TH antibodies were not found in patients with segmental vitiligo, healthy controls and patients with other autoimmune diseases without concomitant vitiligo [54]. Moreover, a study on TH epitopes recognized by TH antibodies in patients with vitiligo and alopecia areata (AA) reported that $\mathrm{TH}$ antibodies from patients with vitiligo or AA can recognize identical epitopes [57].

\section{Tyrosine related protein (TRP-2)}

Tyrosinase and tyrosinase associated protein 1 and protein 2 catalyze the biochemical steps in the biosynthesis of melanin [4]. The TRP2 protects human against oxidative stress by increasing glutathione levels and by reducing the toxicity of quinones and DNA damage induced by free radicals [58]. Several melanosome glycoproteins have been shown to be antigenic in human. A previous study on antibody responses to a melanocyte autoantigen, TRP-2, found that this autoantigen is immunogenic in human [59]. The TRP-2 antibody responses provide a linkage between autoimmune responses by vitiligo and melanoma patients responding to immunotherapy who have induced hypopigmentation [60]. However a more recent study reported that TRP-2 transcripts from peripheral blood mononuclear cells (PBMCs) are absent in vitiligo patients but present in healthy individuals [51]. Considering these findings, studies on Anti-TRP-2 are inconsistent; therefore studies to confirm its reliability as potential biomarker for development of new diagnostic test in vitiligo could be a good approach.

\section{Other Antibodies}

Circulating organ-specific autoantibodies particularly to the thyroid, adrenal glands, gastric parietal cells, and pancreatic islet cells are commonly detected in the sera of vitiligo patients [61]. Moreover, antinuclear antibody and IgM-rheumatoid factor have been detected at a significant frequency in vitiligo patients [32]. Anti-keratinocyte intracellular antibodies that correlate with disease extent and activity have also been detected in vitiligo patients [62]. Tyrosinase-related protein 1 (TYRP1) is a critical enzyme for the correct trafficking of tyrosinase to melanosomes [63]. In addition, autoantibodies against TYP1 are also suggested in vitiligo patients (Table 1).

\section{Conclusion}

It is obvious there is strong indication that autoantibodies are playing significant role in vitiligo pathogenesis. Although there are some disputed findings, however most of the studies indicate the presence of autoantibodies in vitiligo patients, therefore the use of these antibodies for development of new laboratory test for diagnosis is indeed a good approach. The antibodies in vitiligo can serve as potential biomarkers for monitoring and assessment of autoimmune diseases in vitiligo patients. In-depth researches in this area could likely gives a good conclusion on the pathogenic mechanism of vitiligo in autoimmune diseases. Certainly, there are more to discover in vitiligo pathogenesis. 


\section{References}

1. Daneshpazhooh M, Mostofizadeh GM, Behjati J, Akhyani M, Robati RM (2006) Anti-thyroid peroxidase antibody and vitiligo: a controlled study. BMC Dermatol 6: 3 .

2. Richmond JM, Frisoli ML, Harris JE (2013) Innate immune mechanisms in vitiligo: danger from within. Curr Opin Immunol 25: 676-682.

3. Alikhan A, Felsten LM, Daly M, Petronic-Rosic V (2011) Vitiligo: a comprehensive overview Part I. Introduction, epidemiology, quality of life, diagnosis, differentia diagnosis, associations, histopathology, etiology, and work-up. J Am Acad Dermatol 65: 473-91.

4. González R, Torres-López E (2014) Immunological basis of melanomaassociated vitiligo-like depigmentation. Actas Dermosifiliogr 105: 122-127.

5. Taïeb A, Picardo M; VETF Members (2007) The definition and assessment of vitiligo: a consensus report of the Vitiligo European Task Force. Pigment Cell Res 20: 27-35.

6. Taïeb A, Picardo M (2010) Epidemiology, definitions and classification. In Vitiligo, Berlin, Heidelberg: Springer, 13-24.

7. Spritz RA (2010) Shared genetic relationships underlying generalized vitiligo and autoimmune thyroid disease. Thyroid 20: 745-754.

8. Spritz RA (2012) Six decades of vitiligo genetics: genome-wide studies provide insights into autoimmune pathogenesis. J Invest Dermatol 132: 268-273.

9. Mazereeuw-Hautier J, Bezio S, Mahe E, Bodemer C, Eschard C, et al (2010) Segmental and nonsegmental childhood vitiligo has distinct clinical characteristics: a prospective observational study. J Am Acad Dermatol 62 . 945-949.

10. van Geel N, Vandenhaute S, Speeckaert R, Brochez L, Mollet I, et al. (2011) Prognostic value and clinical significance of halo naevi regarding vitiligo. $\mathrm{Br} J$ Dermatol 164: 743-749.

11. Tu C, Zhao D, Lin X (2001) Levels of neuropeptide- $Y$ in the plasma and skin tissue fluids of patients with vitiligo. J Dermatol Sci 27: 178-182.

12. Antony FC, Marsden RA (2003) Vitiligo in association with human immunodeficiency virus infection. J Eur Acad Dermatol Venereol 17: 456-458.

13. Martínez D, Vermeulen M, von Euw E, Sabatté J, Maggíni J, et al. (2007) Extracellular acidosis triggers the maturation of human dendritic cells and the production of IL-12. J Immunol 179: 1950-1959.

14. Nanda A (2008) Autoimmune diseases associated with neurofibromatosis type 1. Pediatr Dermatol 25: 392-393.

15. Cucchi ML, Frattini P, Santagostino G, Preda S, Orecchia G (2003) Catecholamines increase in the urine of non-segmental vitiligo especially during its active phase. Pigment Cell Res 16: 111-116.

16. Natarajan VT, Singh A, Kumar AA, Sharma P, Kar HK, (2010) Transcriptional upregulation of Nrf2-dependent phase II detoxification genes in the involved epidermis of vitiligo vulgaris. J Invest Dermatol 130: 2781-2789.

17. Jian Z, Li K, Liu L, Zhang Y, Zhou Z, et al. (2011) Heme oxygenase-1 protects human melanocytes from $\mathrm{H} 2 \mathrm{O} 2$-induced oxidative stress via the Nrf2-ARE pathway. J Invest Dermatol 131: 1420-1427.

18. Imokawa G, Moretti S (2010) Cytokines and growth factors. In: Vitiligo. Berlin, Heidelberg: Springer, 269- 282.

19. Meyer KC, Brzoska T, Abels C, Paus R (2009) The alpha-melanocyte stimulating hormone-related tripeptide K(D)PT stimulates human hair follicle pigmentation in situ under proinflammatory conditions. Br J Dermatol 160: 433-437.

20. Usha SS, Pandey SS (2009) Role of Autoimmunity in Vitiligo. Indian J Allergy Asthma Immunol 23: 67-71.

21. Waterman EA, Gawkrodger DJ, Watson PF, Weetman AP, Kemp EH (2010) Autoantigens in vitiligo identified by the serological selection of a phagedisplayed melanocyte cDNA expression library. J Invest Dermatol 130: 230 240.

22. Wu CS, Lan CC, Yu HS (2012) Narrow-band UVB irradiation stimulates the migration and functional development of vitiligo-lgG antibodies-treated pigment cells. J Eur Acad Dermatol Venereol 26: 456-464

23. Kemp EH, Gavalas NG, Gawkrodger DJ, Weetman AP (2007) Autoantibody responses to melanocytes in the depigmenting skin disease vitiligo. Autoimmun Rev 6: 138-142.
24. Li Q, LV Y, Li C, Yi X, Long HA, et al. (2011) Vitiligo autoantigen VIT75 is identified as lamin $A$ in vitiligo by serological proteome analysis based on mass spectrometry. J Invest Dermatol 131: 727-734.

25. Raposo G, Marks MS (2007) Melanosomes--dark organelles enlighten endosomal membrane transport. Nat Rev Mol Cell Biol 8: 786-797.

26. Simon JD, Hong L, Peles DN (2008) Insights into melanosomes and melanin from some interesting spatial and temporal properties. J Phys Chem B 112 13201-13217.

27. Tsatmali M, Ancans J, Thody AJ (2002) Melanocyte function and its control by melanocortin peptides. J Histochem Cytochem 50: 125-133.

28. Zailaie MZ (2005) Aspirin reduces serum anti-melanocyte antibodies and soluble interleukin-2 receptors in vitiligo patients. Saudi Med J 26: 1085-1091.

29. Li YL, Yu CL, Yu HS (2000) lgG anti-melanocyte antibodies purified from patients with active vitiligo induce HLA-DR and intercellular adhesion molecule-1 expression and an increase in interleukin- 8 release by melanocytes. $\mathrm{J}$ Invest Dermatol 115: 969-73.

30. van den Boorn JG, Konijnenberg D, Dellemijn TA, van der Veen JP, Bos JD, et al. (2009) Autoimmune destruction of skin melanocytes by perilesional T cells from vitiligo patients. J Invest Dermatol 129: 2220-2232.

31. van den Wijngaard R, Wankowicz-Kalinska A, Le Poole C, Tigges B, Westerho $W$ et al, (2000) Local immune response in skin of generalized vitiligo patients. Destruction of melanocytes is associated with the prominent presence of CLA T cells at the perilesional site. Lab Invest 80: 1299-309.

32. Farrokhi S, Hojjat-Farsangi M, Noohpisheh MK, Tahmasbi R, Rezaei N (2005) Assessment of the immune system in 55 Iranian patients with vitiligo. $\mathrm{J}$ Eur Acad Dermatol Venereol 19: 706-711.

33. Kemp EH, Emhemad S, Gawkrodger DJ, Weetman AP (2011) Autoimmunity in vitiligo In : Mavragani C (1 ${ }^{\text {st }}$ Edn.), Autoimmune Disorders - Pathogenic Aspects, Rijeka, InTech, Croatia, 271-294

34. McLachlan SM, Rapoport B (2000) Autoimmune response to the thyroid in humans: thyroid peroxidase--the common autoantigenic denominator. Int Rev Immunol 19: 587-618.

35. TrbojeviÄ $\ddagger$ B, Djurica S (2005) [Diagnosis of autoimmune thyroid disease]. Srp Arh Celok Lek 133 Suppl 1: 25-33.

36. Betterle C, Del Prete GF, Peserico A, Bersani G, Caracciolo F, et al. (1976) Autoantibodies in vitiligo. Arch Dermatol 112: 1328

37. Dave S, D'souza M, Thappa DM, Reddy KS, Bobby Z, (2003) High frequency of thyroid dysfunction in Indian patients with vitiligo. Indian Journal of Dermatology 48: 68-72.

38. Vrijman C, Kroon MW, Limpens J, Leeflang MM, Luiten RM, et al. (2012) The prevalence of thyroid disease in patients with vitiligo: a systematic review. $\mathrm{Br} \mathrm{J}$ Dermatol 167: 1224-1235.

39. Kasumagic-Halilovic E, Ovcina-Kurtovic N, Jukic T, Karamehic J, Begovic B, et al. (2013) Vitiligo and autoimmunity. Med Arch 67: 91-93.

40. Brzoska T, Luger TA, Maaser C, Abels C, Bohm M (2008) Alpha-melanocytestimulating hormone and related tripeptides: biochemistry, anti-inflammatory and protective effects in vitro and in vivo, and future perspectives for the treatment of immune-mediated inflammatory diseases. Endocr Rev 29: 581 602 .

41. Brenner M, Hearing VJ (2008) Modifying skin pigmentation - approaches through intrinsic biochemistry and exogenous agents. Drug Discov Today Dis Mech 5: 189-199.

42. Pichler R, Sfetsos K, Badics B, Gutenbrunner S, Aubock J (2006) Vitiligo patients present lower plasma levels of alpha-melanotropin immunoreactivities. Neuropeptides 40: 177-783.

43. Wakamatsu K, Graham A, Cook D, Thody AJ (1997) Characterisation of ACTH peptides in human skin and their activation of the melanocortin-1 receptor Pigment Cell Res 10: 288-297.

44. Graham A, Westerhof W, Thody AJ (1999) The expression of alpha-MSH by melanocytes is reduced in vitiligo. Ann N Y Acad Sci 885: 470-473.

45. Agretti P, De Marco G, Sansone D, Betterle C, Coco G, et al, (2010) Patients affected by vitiligo and autoimmune diseases do not show antibodies interfering with the activity of the melanocortin 1 receptor. J Endocrinol Invest 33: 784-788.

46. Gavalas NG, Gottumukkala RV, Gawkrodger DJ, Watson PF, Weetman AP 
(2009) Mapping of melanin-concentrating hormone receptor $1 \mathrm{~B}$ cell epitopes predicts two major binding sites for vitiligo patient autoantibodies. Exp Dermato 18: $454-463$

47. Gottumukkala RV, Gavalas NG, Akhtar S, Metcalfe RA, Gawkrodger DJ, et al. (2006) Function-blocking autoantibodies to the melanin-concentrating hormone receptor in vitiligo patients. Lab Invest 86: 781-789.

48. Hoogduijn MJ, Ancans J, Suzuki I, Estdale S, Thody AJ (2002) Melaninconcentrating hormone and its receptor are expressed and functional in human skin. Biochem Biophys Res Commun 296: 698-701.

49. Kemp EH, Waterman EA, Hawes BE, O'Neill K, Gottumukkala RV, et al. (2002) The melanin-concentrating hormone receptor 1 , a novel target of autoantibody responses in vitiligo. J Clin Invest 109: 923-930.

50. Chen JP, Li HP, Jin SH, Zhang JT, Li J (2009) Detection of serum autoantibodies to melanocyte and correlation between melanoma antigen recognized by T-cells and vitiligo in children. Nan Fang Yi Ke Da Xue Xue Bao 29: 2107-2108.

51. Bam M, Bagchi T (2009) MART-1 transcript is absent in PBMCs from Vitiligo patients. Central European Journal of Biology 4: 528-535.

52. Nesher G, Margalit R, Ashkenazi YJ (2001) Anti-nuclear envelope antibodies: Clinical associations. Semin Arthritis Rheum 30: 313-320.

53. Deo SS, Bhagat AR, Shah RN (2011) Identification of Autoantibody to Melanocytes and Characterization of Different Autoantigens in Western Indian Vitiligo Patients. J Clin Exp Dermatol Res 2: 120-122.

54. Kemp EH, Emhemad S, Akhtar S, Watson PF, Gawkrodger DJ, et al. (2011) Autoantibodies against tyrosine hydroxylase in patients with non-segmental (generalised) vitiligo. Exp Dermatol 20: 35-40.

55. Marles LK, Peters EM, Tobin DJ, Hibberts NA, Schallreuter KU (2003) Tyrosine hydroxylase isoenzyme I is present in human melanosomes: a possible novel function in pigmentation. Exp Dermatol 12: 61-70.

56. Kågedal B, Kullman A, Lenner L, Träger C, Kogner P, et al. (2004) Pterindependent tyrosine hydroxylase mRNA is not expressed in human melanocytes or melanoma cells. Pigment Cell Res 17: 346-351.

57. Rahoma SF, Sandhu HK, McDonagh AJ, Gawkrodger DJ, Weetman AP, et al (2012) Epitopes, avidity and IgG subclasses of tyrosine hydroxylase autoantibodies in vitiligo and alopecia areata patients. Br J Dermatol 167: 17-28.
58. Michard Q, Commo S, Belaidi JP, Alleaume AM, Michelet JF, et al. (2008) TRP 2 specifically decreases WM35 cell sensitivity to oxidative stress. Free Radic Biol Med 44: 1023-1031.

59. Denat L, Kadekaro AL, Marrot L, Leachman SA, Abdel-Malek ZA (2014) Melanocytes as instigators and victims of oxidative stress. J Invest Dermatol 134: 1512-1518.

60. Okamoto T, Irie RF, Fujii S, Huang SK, Nizze AJ, et al, (1998) Anti-tyrosinaserelated protein-2 immune response in vitiligo patients and melanoma patients receiving active-specific immunotherapy. J Invest Dermatol 111: 1034-1039.

61. Mandry RC, Ortíz LJ, Lugo-Somolinos A, Sánchez JL (1996) Organ-specific autoantibodies in vitiligo patients and their relatives. Int J Dermatol 35: 18-21.

62. Yu HS, Kao CH, Yu CL (1993) Coexistence and relationship of antikeratinocyte and antimelanocyte antibodies in patients with non-segmental-type vitiligo. $\mathrm{J}$ Invest Dermatol 100: 823-828.

63. Toyofuku K, Wada I, Valencia JC, Kushimoto T, Ferrans VJ, et al, (2001) Oculocutaneous albinism types 1 and 3 are ER retention diseases: mutation of tyrosinase or Tyrp1 can affect the processing of both mutant and wild-type proteins. FASEB J 15: 2149-2161.

64. Brostoff J (1969) Autoantibodies in patients with vitiligo. Lancet 2: 177-178.

65. Kemp EH, Gawkrodger DJ, MacNeil S, Watson PF, Weetman AP (1997) Detection of tyrosinase autoantibodies in patients with vitiligo using 35S-labeled recombinant human tyrosinase in a radioimmunoassay. J Invest Dermatol 109 69-73.

66. Zhou M, Guan C, Lin F, Xu W, Fu L, et al. (2011) Immunodetection of the MCHR1 antibody in vitiligo patient sera. Int J Mol Med 27: 725-729.

67. Waterman EA, Kemp EH, Gawkrodger DJ, Watson PF, Weetman AP (2002) Autoantibodies in vitiligo patients are not directed to the melanocyte differentiation antigen MelanA/MART1. Clin Exp Immunol 129: 527-532.

68. Kim JY, Do JE, Ahn KJ, Noh S, Jee HJ, et al. (2011) Detection of melanocyte autoantigens reacting with autoantibodies in vitiligo patients by proteomics. $J$ Dermatol Sci 62: 202-204. 